\title{
Pre-indexing Pruning Strategies
}

\author{
Soner Altin ${ }^{1}[0000-0003-3896-6559]$ \\ Ricardo Baeza-Yates $2,1[0000-0003-3208-9778]$ \\ B. Barla Cambazoglu ${ }^{3[0000-0003-2192-3819]}$ \\ 1 Web Science \& Social Computing Research Group \\ DTIC, Universitat Pompeu Fabra, Barcelona, Spain \\ ${ }^{2}$ Khoury College of Computer Sciences \\ Northeastern University at Silicon Valley, USA \\ 3 RMIT University, Melbourne, Australia
}

\begin{abstract}
We explore different techniques for pruning an inverted index in advance, that is, without building the full index. These techniques provide interesting trade-offs between index size, answer quality and query coverage. We experimentally analyze them in a large public web collection with two different query logs. The trade-offs that we find range from an index of size $4 \%$ and $35 \%$ of precision@10 to an index of size $46 \%$ and $90 \%$ of precision@10, with respect to the full index case. In both cases we cover almost $97 \%$ of the query volume. We also do a relative relevance analysis with a smaller private web collection and query log, finding that some of our techniques allow a reduction of almost $40 \%$ the index size by losing less than $2 \%$ for NDCG@10.
\end{abstract}

Keywords: Web search · inverted index · index pruning · search efficiency.

\section{Introduction}

Commercial web search engines evaluate queries by processing a very large inverted index built using pages crawled from the Web. Storing and maintaining such an index as well as providing low-latency query processing requires a large amount of hardware investments. Therefore, performance optimization in the context of web search engine indexes has been a very active research area in the last couple of decades.

Among the possible optimizations, a relatively well studied one is static index pruning. The main idea behind this optimization is to create a so-called pruned inverted index which stores less information than the full web index while attaining the search result quality obtained with a full index as much as possible. A pruned index has lower space requirements and leads to faster query processing since fewer postings are stored and processed. The main challenge is to prevent degradation in search quality and query coverage due to the absence of potentially useful indexed content in the pruned index.

All existing approaches so far assume the presence of a full web index to facilitate the construction of the pruned index. That is, a pruned index is created by removing postings from the inverted lists in the full web index, entirely or 


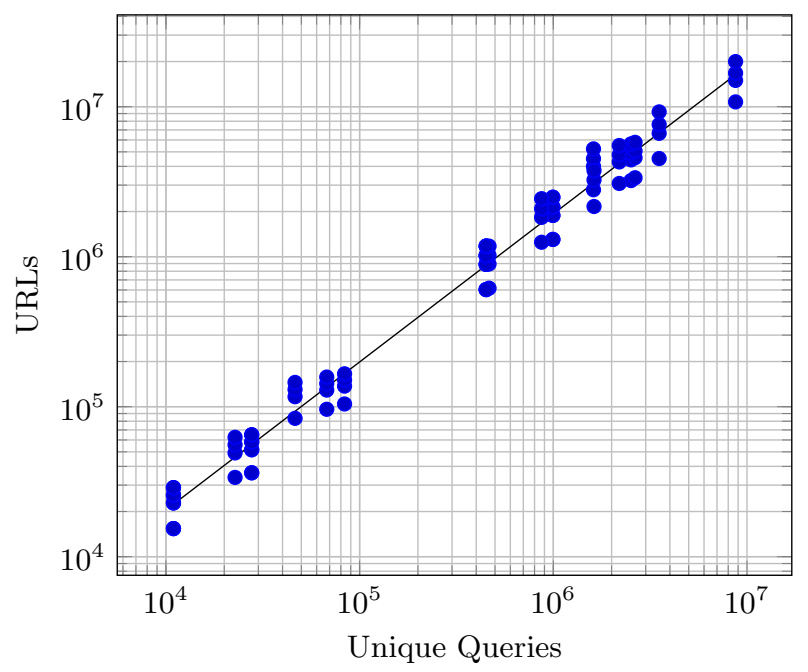

Fig. 1. Relation between unique URLs and unique queries and the simple model found.

selectively, by using statistical techniques, with the objective of maintaining the search quality. In certain scenarios, however, the resource constraints are really tight (e.g., a low-budget web search engine), and it may not be feasible to build and/or store the full web index. Therefore, pruning decisions need to be made without constructing a full web index first, perhaps even at crawling time to reduce the storage requirements. Hence, how much we lose if we decide a priori which documents or parts of them should be indexed?

Another motivation comes from [4], that studied the minimal set of URLs $U$ from a web inverted index that are needed to cover a maximal number of unique queries. They consider that a query is covered if at least one clicked top- $k$ result for that query belongs to $U$ (for $k$ varying from 10 to 1,000). To find $U$ they only used queries with a minimal frequency ranging from 5 to 50 occurrences and pairs (query, clicked result) that appeared at least 5 to 20 times in a period ranging from one day to six months. This setup generated 91 different data sets reaching up to 10 million queries and 50 million URLs. Using these data sets characteristics we can find a simple model between the relation of unique queries and unique clicked URLs given by $U=2.308 Q^{0.987}$ with $R=0.986$ where $U$ is the number of unique URLs and $Q$ is the number of unique queries (see Figure 1).

Although the model is simple, the error is low, and says that $U$ grows almost linearly with $Q$, but for all practical purposes, always there are more URLs than queries (e.g., for 1B unique queries we have 1.76B unique clicked URLs). As unique long tail queries grow, this implies that the index grows even faster. Hence, it makes sense to select documents that cover more queries than others, because as query coverage follows a power law, with a small fraction of documents we can cover an even larger fraction of queries. This is even more important in the long tail as the coverage there is lower. Indeed, in [4] they found that $0.7 \%$ of the web pages covered $25 \%$ of the queries. Although this result was for a static set 
of queries, in [5] they found that the correlation of query terms for segments of 3 weeks across a period of 15 weeks was above 0.995 . That is, query distribution changes slowly in time. On the other hand, the former result can only be found if the whole index is available. Hence, can a similar result be achieved without having the full index?

Motivated by the aforementioned scenarios, here we design document pruning strategies which do not require the presence of a full web index. That is, the pruning decisions are made in a document-centric manner, i.e., the indexed content is determined as the documents flow in the ingestion pipeline (the sequence of software modules that convert crawled documents to an indexable form). We propose various heuristics to exclude documents' content, partially or entirely, from the index based on the size of the documents, their query-independent summaries, and query terms statistics.

We perform experiments using a public web collection of 50M pages and a private web collection of $18 \mathrm{M}$ pages as well as three different search query logs, one public and two private. As main evaluation metrics, we report the reduction attained in the index size, the loss in search quality with respect to a search system relying on a full web index as well as the unique and overall query coverage. To measure search quality we use precision@10 and NDCG@10 with respect to the full index.

The obtained results are encouraging as the index size can be significantly reduced without hurting the search quality too much and without having to build the full index. The trade-offs that we find range from an index of size $4 \%$ and $35 \%$ of precision@10 to an index of size $46 \%$ and $90 \%$ of precision@10, with respect to the full index case. In both cases we cover $83 \%$ of all unique queries and almost $97 \%$ of the query volume. In the case of NDCG@10, some techniques loose less than $2 \%$ providing almost $40 \%$ reduction in the index size.

Summarizing, our contributions are two fold: (1) we introduce a new pruning method based in summarization, which performs better than most traditional pruning methods (detailed in Section 3.2); and (2) we perform a thorough experimental comparison of all known pre-indexing pruning techniques.

The rest of the paper is organized as follows. Section 2 gives an overview of the related work. The pruning heuristics are described in Section 3. The experimental setup is explained in Section 4 while the results of the experiments are presented in Section 5. Section 6 gives our conclusions and future work.

\section{Related Work}

Unlike this work, which assumes that the content of the documents are pruned (partially or entirely) without constructing a full index, a number of works in literature considered making the pruning decisions after constructing such an index. This line of research is known as static index pruning. The earliest work belongs to Carmel et al. [12], who adopted a term pruning approach where an inverted list is entirely removed from the full index if the score contribution of the corresponding term is lower than a certain threshold. Three different strate- 
gies are evaluated to determine the threshold: a uniform thresholding strategy that applies to all list entries and two others, list-specific thresholding strategies. De Moura et al. [14] argue that the techniques used by Carmel et al. [12] are not very effective when queries are processed in conjunctive mode or contain phrases. To alleviate this issue, they exploit the occurrence of terms in important sentences in pages. A page entry is preserved in the pruned index only if the respective term for the list appears in at least one of the important sentences of the page. Büttcher et al. [10] use a language model to determine each page's most important terms and keep them in the pruned index. Blanco and Barreiro [8] prune entire inverted lists based on the informativeness of their corresponding terms. Ntoulas et al. [17] evaluate three different approaches, where pruned items are entire inverted lists, all inverted list entries belonging to a page, or selected inverted list entries only. Some of those approaches provide correctness guarantees, i.e., the search results obtained by evaluating the query on the pruned index are identical to those that would be obtained from the full index. Blanco and Barreiro [9] apply the probability ranking principle to static index pruning. Thota et al. [21] exploit the entropy measure to come up with a document-centric pruning approach. Altingovde et al. [1] make use of term and page access statistics in query logs to guide the pruning process. In a relatively recent study, Chen and Lee [13] argue that the earliest pruning approach, proposed by Carmel et al. [12], is superior to other pruning approaches in web-scale settings. Skobeltsyn et al. [20] investigate the interplay between search result caching and static index pruning.

Apart from static index pruning, there are other architectural optimizations, such as tiering $[19,6,16]$ and selective search $[3,18,15]$. Another line of research with a vast number of works includes dynamic index pruning, where the inverted lists are pruned at query processing time. We do not cover them here as they are orthogonal to static index pruning. A good survey of efficiency optimizations related to search engines can be found in [11].

Other related problems include document selection in federated search and other types of distributed search (see Chapter 10 of [7]).

\section{Pruning Heuristics}

Based on the granularity at which document content is pruned, we propose three types of heuristics: document-, sentence, and term-level pruning. Document-level pruning heuristics include or exclude the entire document without investigating its constituting syntactical units. Sentence-level heuristics select a (potentially empty) set of sentences to be pruned from the document content. Finally, termlevel heuristics deal with individual terms statistics taken from query logs or consider removing/transforming specific sets of words, providing the finest granularity of pruning. All the strategies presented in this section are summarized in Table 1. 


\subsection{Document-Level Pruning}

Document size Very large documents may take too much space in the index. Yet, their contribution to search quality is often not likely to be very different from medium-size documents. Based on this idea, we exclude largest $L \%$ of documents in the collection from the index and we index the remaining documents. The size of a document is determined based on the number of bytes in the textual content that remains after removing the document's boilerplate.

Set cover Documents vary in their likelihood of being a good match to a web query. Also, indexing certain documents is relatively more important as they may match many queries, while certain documents are relatively less important since they are seldom queried. The set cover heuristic which was previously proposed in [2] exploits this idea. Essentially, this heuristic tries to select a minimal subset $D$ of documents from a given collection such that the number of queries whose ideal top $k$ results (obtained using a full web index) contain at least one document from $D$ is maximized. In other words, this heuristic tries to minimize the number of queries whose top-k results (obtained using a pruned index) do not contain any ideal results. Once we find a set cover $D$, we index all documents in $D$.

Access-based Document-Centric Pruning Altingovde et al. [1] proposed an access based pruning strategy to prune documents directly from the collection. Documents with low access count are removed from the collection until a fraction of pruned documents is reached.

\subsection{Sentence-Level Pruning}

Summarization Certain sentences in a document are relatively more important or represent the document better than the others. It may be more beneficial to index such sentences as they are more likely to be of interest to users and match their queries. To this end, we apply the Textteaser summarizer ${ }^{4}$ to the content of documents to obtain the most important or representative $S$ sentences and then index only the terms occurring in those sentences. If the document contains less than $S$ sentences, we do not apply summarization, i.e., we index all of the terms in the document.

\subsection{Term-Level Pruning}

Query terms popularity It is usually important to index terms that often appear in web queries. In this heuristic, we extract the set of terms occurring in a web query $\log$ to obtain a representative set of such useful terms. When processing documents, we index only the terms which appear in this set.

Stopword removal and stemming We also considered these standard term processing operations as baseline cases for term-level pruning. We have three cases, just stopwords, just stemming, or use both of them.

\footnotetext{
${ }^{4}$ https://github.com/MojoJolo/textteaser.
} 
Table 1. Properties of the heuristics.

\begin{tabular}{lcc|lcc}
\hline Index & \multicolumn{2}{c}{$\begin{array}{c}\text { Uses training } \\
\text { queries, documents }\end{array}$} & Index & \multicolumn{2}{c}{$\begin{array}{c}\text { Uses training } \\
\text { queries, documents }\end{array}$} \\
\hline \hline Full & No & No & TermPopularity & Yes & No \\
\hline DocumentSize & No & No & Stemming & No & No \\
SetCover & Yes & Yes & Stopwords & No & No \\
aDCP & Yes & Yes & StemStop & No & No \\
\cline { 1 - 2 } Summarization & No & No & & &
\end{tabular}

\section{Experimental Setup}

\subsection{Document Collection}

As web document collection, we mostly use the open source web collection provided by Common Crawl, CC, in November 2017. ${ }^{5}$ In total, the CC collection takes $4 \mathrm{~TB}$ of disk space when compressed. The full web index constructed using this collection contains 50.3M English documents (see end of next section). The average document size is $3.5 \mathrm{~KB}$ before parsing the HTML content, and after processing the average number of sentences and terms in a document is around 26 and 628, respectively. This collection is quite diverse in that it contains pages crawled from more than $1.3 \mathrm{M}$ different web domains.

For the relevance evaluation we use an ad-hoc web collection A of almost $19 \mathrm{M}$ English web documents, also crawled in 2017. This collection is even more diverse as it contains content from $1.6 \mathrm{M}$ different domains.

\subsection{Document Processing}

Before indexing, we have a document processing pipeline that includes HTML parsing, boilerplate extraction, sentence extraction, summarization, tokenization, and language detection. For consistency, we apply the same pipeline to documents and queries.

We first use the open-source Boilerpipe library to remove the boilerplate of the web document (e.g., headers, footers, menus, and ads in the document). ${ }^{6}$ Accurate removal of the boilerplate is important since failure to remove the boilerplate may affect the quality of the succeeding text processing steps, such as language detection or summarization. We then use the Jsoup library to extract the textual content of the document from the remaining HTML content as well as the document's title. For sentence extraction and tokenization, we use OpenNLP's English models. ${ }^{7}$ To identify the language of a document, we use Fasttext's open-source language detection model, which has support for 176

\footnotetext{
${ }^{5}$ Common Crawl web collection, http://commoncrawl.org/2017/11/ november-2017-crawl-archive-now-available/

${ }^{6}$ Boilerpipe, https://github.com/robbypond/boilerpipe.

${ }^{7}$ http://opennlp.sourceforge.net/models-1.5/
} 
languages. ${ }^{8}$ We use only documents whose language is detected as English and when the likelihood estimated by the model is larger than 0.8 .

\subsection{Indexing}

We index documents using an open source version of Elasticsearch. ${ }^{9}$ The documents are indexed in an incremental fashion. All indexes were created with 15 shards with a replication factor of 3. Full is the full index constructed using the entire collection for comparison purposes. Most of the evaluation metrics computed are relative to this index.

We used Elasticsearch's built-in stopword remover and its built-in stemmer, which removes only possessive suffixes, to obtain our three baselines: Stemming, Stopwords, and StemStop). In all other cases, we do not perform stopword removal nor stemming.

For the DocumentSize heuristic, we set $L$ to $1 \%$ and $10 \%$. These thresholds result in pruning of documents that are larger than $7 \mathrm{~KB}$ and $33 \mathrm{~KB}$, respectively. In the SetCover heuristic, we set the $C$ parameter to 1,5 , or 10 , resulting in approximately $3.1,1.7$ and 1.3 million documents being indexed, respectively. In the Summarization heuristic, we set the $S$ parameter to $10,20,40,80$, and 160 . For the aDCP heuristic, we set the $\mu$ parameter to $0.1,0.2$ and 0.3 resulting in 5 , 10 and 15 million documents being indexed, respectively. In the case of the Term Popularity index, we prune all terms which do not appear in a given query log.

Table 2 summarizes the size properties of the constructed indexes.

\subsection{Ranking}

We use two different ranking techniques in the experimental comparison. The simplest one just uses the well-known BM25 technique (see [7], Section 3.5.1) which is native to ElasticSearch, as it is one of the best baselines based just in textual content. A more sophisticated version uses a two phase ranking approach, first using BM25 to obtain a pool of 2,000 candidates and then using learningto-rank (LTR), LambdaMart [22], to do the final ranking. The LTR variant uses more than 200 features that include query-document similarity (44\%), link analysis (20\%), query-document relevance (16\%), URL name features (10\%) and textual content $(10 \%)$ features.

\subsection{Query Logs}

In most experiments, we use a public query $\log \mathrm{A}$, which contains 7.3 million queries submitted in 2006. The query log is split, in temporal order, into training and test sets, which contain 6.4 million and 900K queries, respectively. The training set is used to compute the set cover as well as terms' query frequencies. The test queries are used for evaluation.

\footnotetext{
${ }^{8}$ https://fasttext.cc/docs/en/language-identification.html.

9 https://www.elastic.co/products/elasticsearch
} 
Table 2. Index sizes and corresponding pruning ratios $(P R)$ shown as percentages.

\begin{tabular}{|c|c|c|c|}
\hline Index & \multicolumn{3}{|c|}{ Parameter Size (GB) $P R(\%)$} \\
\hline Full & - & 180.200 & 0.00 \\
\hline DocumentSize (DS\#) & $L=1 \%$ & 54.147 & 69.95 \\
\hline DocumentSize & $L=10 \%$ & 32.226 & 82.12 \\
\hline SetCover (SC\#) & $C=1$ & 9.400 & 94.78 \\
\hline etCover & $C=5$ & 7.000 & 96.12 \\
\hline etCover & $C=10$ & 6.600 & 96.34 \\
\hline $\mathrm{aDCP}(\mathrm{aDECP} \#)$ & $\mu=0.1$ & 22.900 & 87.29 \\
\hline $\mathrm{aDCP}$ & $\mu=0.2$ & 31.600 & 81.90 \\
\hline $\mathrm{aDCP}$ & $\mu=0.3$ & 41.100 & 77.19 \\
\hline$\overline{\text { Summarization (S\#) }}$ & $S=10$ & 23.725 & $\overline{86.83}$ \\
\hline ummarization & $S=20$ & 32.930 & 81.73 \\
\hline Summarization & $S=40$ & 42.526 & 76.40 \\
\hline Summarization & $S=80$ & 50.713 & 71.86 \\
\hline Summarization & $S=160$ & 82.58 & 54.17 \\
\hline TermPopularity (TP) & - & 52.014 & 71.14 \\
\hline Stemming $(\mathrm{ST})$ & - & 69.791 & 61.27 \\
\hline Stopwords (SW) & - & 64.338 & 64.30 \\
\hline StemStop (BOTH) & - & 60.907 & 66.20 \\
\hline
\end{tabular}

To measure the relevance of the results as well as the temporal robustness of them, we also use two newer (2017) but smaller query logs, B1 and B2, obtained from a commercial search engine. Query log B1 contains about 300K queries with relevance judgments for the top 50 results of our LTR model, where $125 \mathrm{~K}$ queries are used for training the model and the $175 \mathrm{~K}$ others are left for relevance evaluation. Query $\log \mathrm{B} 2$ contains almost 2.7M queries and they are used to study how sensitive are our pruning techniques to a query log from a different search engine and from a different time (notice that the year of this query log matches the year of the web collection).

\subsection{Evaluation}

To evaluate the proposed pruning strategies, we use the Elasticsearch API to submit each of the two test sets of queries sequentially, to compute the metrics detailed below. In all experiments, we set $k$ to 100, unless otherwise stated.

Pruning ratio ( $P R$ ) For each index, we compute the ratio of its size to the size of the Full index (in bytes) and subtract this ratio from 1 as follows $P R=$

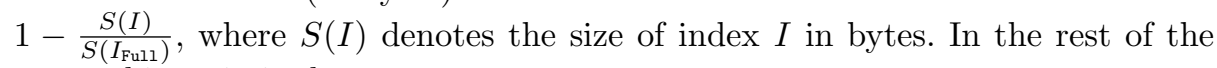
paper this ratio is shown as a percentage.

Average precision ( $A P @ k$ ) For each query, we compute the precision as the fraction of relevant results within the retrieved top- $k$ results set. We assume that the top- $k$ results obtained by processing the full web index constitute our relevant results. We then average the precision values over all queries (see Table 3, 
Table 3. Evaluation metrics for query relevance and similarity.

\begin{tabular}{|c|c|c|}
\hline$A P @ k$ & $A R @ k$ & $A R S @ k$ \\
\hline$\frac{\sum_{q=1}^{m} \frac{R_{q}(I, k) \cap R_{q}\left(I_{\mathrm{Ful1}}, k\right)}{R_{q}(I, k)}}{m}$ & $\frac{\sum_{q=1}^{m} \frac{R_{q}(I, k) \cap R_{q}\left(I_{\mathrm{Ful1}}, k\right)}{R_{q}\left(I_{\mathrm{Ful1}}, k\right)},}{m}$ & $\frac{\sum_{q=1}^{m} \frac{\left|R_{q}(I, k) \cap R_{q}\left(I_{\mathrm{Full}}, k\right)\right|}{\left|R_{q}(I, k) \cup R_{q}\left(I_{\mathrm{Fu} 11}, k\right)\right|}}{m}$ \\
\hline
\end{tabular}

first column) where $R_{q}(I, k)$ is the set of top- $k$ retrieved results from the pruned index $I$ for a given query $q . I_{\text {Full }}$ is the full web index and $m$ is the number of queries in the test query sample.

Average recall (AR@k) For each query, we compute the recall as the fraction of retrieved relevant results (full index case) and then average them over all queries (see Table 3, second column).

Average result similarity ( $A R S @ k$ ) This metric measures the similarity of result sets retrieved for all the queries in the test set between the pruned index and the full index, using the Jaccard similarity metric (see Table 3, third column).

Query coverage ( $Q C @ k)$ This is the ratio between the number of unique test queries which have at least one relevant result in its top- $k$ results set and the total number of unique queries in the test set, $Q C @ k=\frac{u}{U}$, where $u$ and $U$ are the number of unique queries that are covered and the total number of unique queries in the test set, respectively.

Query volume coverage ( $Q V C @ k)$ This is the ratio between the number of test queries which have at least one relevant result in its top- $k$ results set and the total number of queries in the test, $Q V C @ k=\frac{v}{V}$, where $v$ and $V$ denote the volume of queries that are covered and the total volume of queries in the test set, respectively.

Normalized discounted cumulative gain (NDCG) NDCG is the most used measure to evaluate relevance for web search (see [7], Section 4.3.4) and we measure it for the first ten results, NDCG@10.

\section{Experimental Results}

\subsection{Common Crawl and BM25}

In this section we present most of the evaluation measures using the CC collection with the BM25 baseline ranking for query logs A and C.

Pruning ratio Table 2 shows the size of each index we created and the corresponding pruning ratios. According to this table, the most aggressive pruning strategy is the SetCover strategy, which prunes $96 \%$ of the full web index, when $C=10$. Even when $C$ is set to 1 , the pruning ratio remains around $95 \%$ with this strategy. The remaining strategies are relatively less aggressive and the pruning ratio remains over $60 \%$ (excluding the Summarization strategy with $S=160$ ). 
Table 4. Evaluation metrics in percentages (CC collection, BM25, query log A).

\begin{tabular}{|c|c|c|c|c|c|c|c|}
\hline Index & Paramet & $P R$ & \multicolumn{5}{|c|}{$A P @ k A R @ k A R S @ k Q C @ k Q V C @ k$} \\
\hline$\overline{\text { Full }}$ & & & 100 & 100 & 100 & 82.91 & 96.54 \\
\hline DocumentSize & $L=1 \%$ & 69.95 & 78.91 & 94.59 & 75.21 & 78.56 & 95.66 \\
\hline ocumentSize & $L=10 \%$ & 82.12 & 58.84 & 87.27 & 51.76 & 72.54 & 94.19 \\
\hline SetCover & $C=1$ & 94.78 & 41.95 & 51.99 & 34.07 & 82.80 & 96.53 \\
\hline etCover & $C=5$ & 96.12 & 36.27 & 47.46 & 29.63 & 82.79 & 96.52 \\
\hline SetCover & $C=10$ & 96.34 & 35.34 & 46.62 & 28.99 & 82.78 & 96.52 \\
\hline $\mathrm{DCP}$ & $\mu=0.1$ & 87.29 & 51.92 & 53.19 & 40.53 & 82.15 & 96.15 \\
\hline $\mathrm{aDCP}$ & $\mu=0.2$ & 81.90 & 63.32 & 53.86 & 51.52 & 82.52 & 96.33 \\
\hline $\mathrm{aDCP}$ & $\mu=0.3$ & 77.19 & 64.97 & 64.09 & 52.28 & 82.69 & 96.43 \\
\hline Summarization & $S=10$ & 86.83 & 39.99 & 65.94 & 31.31 & 70.90 & 93.57 \\
\hline ummarization & $S=20$ & 81.73 & 52.01 & 75.19 & 43.14 & 73.81 & 94.46 \\
\hline Summarization & $S=40$ & 76.40 & 64.27 & 83.91 & 56.64 & 76.21 & 95.11 \\
\hline Summarization & $S=80$ & 71.86 & 73.83 & 90.22 & 68.22 & 78.08 & 95.54 \\
\hline Summarization & $S=160$ & 54.17 & 89.75 & 97.21 & 87.90 & 82.91 & 96.54 \\
\hline TermPopularity & & 71.14 & 71.57 & 79.85 & 61.39 & 79.69 & 95.60 \\
\hline Stemming & & 61.27 & 66.13 & 58.42 & 49.94 & 85.92 & 97.13 \\
\hline Stopwords & & 64.30 & 93.70 & 93.64 & 88.86 & 82.91 & 96.55 \\
\hline StemStop & & 66.20 & 64.47 & 56.73 & 47.64 & 85.92 & 97.13 \\
\hline
\end{tabular}

Table 5. Evaluation metrics in percentages (CC collection, BM25, query log B2).

\begin{tabular}{|c|c|c|c|c|c|c|c|}
\hline \multirow{2}{*}{$\begin{array}{l}\text { Index } \\
\text { Full }\end{array}$} & \multicolumn{2}{|c|}{ Parameter $P R$} & \multicolumn{5}{|c|}{$A P @ k A R @ k A R S @ k Q C @ k Q V C @ k$} \\
\hline & & & 100 & 100 & 100 & 73.43 & 78.09 \\
\hline$\overline{\text { DocumentSize }}$ & $L=1 \%$ & 69.95 & 75.16 & 81.36 & 69.03 & 67.39 & 75.96 \\
\hline DocumentSize & $L=10 \%$ & 82.12 & 56.05 & 66.86 & 45.99 & 59.81 & 72.89 \\
\hline SetCover & $C=1$ & 94.78 & 44.39 & 47.82 & 34.08 & 72.15 & 77.41 \\
\hline SetCover & $C=5$ & 96.12 & 40.02 & 43.94 & 30.55 & 71.97 & 77.27 \\
\hline SetCover & $C=10$ & 96.34 & 39.33 & 43.31 & 30.10 & 71.95 & 77.24 \\
\hline $\mathrm{aDCP}$ & $\mu=0.1$ & 87.29 & 54.53 & 55.51 & 43.65 & 72.92 & 77.79 \\
\hline $\mathrm{aDCP}$ & $\mu=0.2$ & 81.90 & 63.97 & 64.37 & 52.73 & 73.15 & 77.92 \\
\hline $\mathrm{aDCP}$ & $\mu=0.3$ & 77.19 & 64.69 & 64.78 & 53.59 & 73.27 & 77.99 \\
\hline Summarization & $S=10$ & 86.83 & 41.61 & 51.01 & 30.91 & 59.37 & 72.58 \\
\hline Summarization & $S=20$ & 81.73 & 51.48 & 59.92 & 40.55 & 62.16 & 73.73 \\
\hline Summarization & $S=40$ & 76.40 & 61.87 & 69.33 & 52.01 & 64.74 & 74.75 \\
\hline Summarization & $S=80$ & 71.86 & 70.44 & 76.98 & 62.53 & 67.01 & 75.66 \\
\hline Summarization & $S=160$ & 54.17 & 84.75 & 84.39 & 78.82 & 73.43 & 78.09 \\
\hline TermPopularity & & 71.14 & 69.18 & 73.12 & 58.17 & 69.21 & 75.80 \\
\hline Stemming & & 61.27 & 63.97 & 60.49 & 50.91 & 77.14 & 79.45 \\
\hline Stopwords & & 64.30 & 90.56 & 90.51 & 84.65 & 73.44 & 78.09 \\
\hline StemStop & & 66.20 & 61.55 & 58.04 & 47.79 & 77.15 & 79.45 \\
\hline
\end{tabular}

Precision, recall, and result similarity As expected, less aggressive pruning strategies tend to yield the highest precision values. For example, with query $\log \mathrm{A}$ (Table 4), the highest precision value (90\%) is obtained using the Summarization strategy with $S=160$, while the lowest value $(35 \%)$ is obtained using the 
Table 6. Relevance results (collection A, LTR, query log B2).

\begin{tabular}{|c|c|c|c|c|c|}
\hline Index & Parameter & Size (GB) & $P R(\%)$ & $N D C G @ 10(\%)$ & $\Delta N D C G @ 10(\%)$ \\
\hline Full & & 172.1 & 0.00 & 100 & 0 \\
\hline Summarization & $S=40$ & 133.9 & 22.19 & 98.97 & -1.02 \\
\hline Summarization & $S=20$ & 119.6 & 30.50 & 98.63 & -1.36 \\
\hline Summarization & $S=10$ & 107.5 & 37.53 & 98.46 & -1.53 \\
\hline $\mathrm{aDCP}$ & $\mu=0.3$ & 47.2 & 72.57 & 79.59 & -20.41 \\
\hline DocumentSize & $L=1 \%$ & 145.6 & 15.39 & 69.98 & -30.02 \\
\hline TermPopularity & & 95.4 & 44.56 & 30.21 & -69.79 \\
\hline SetCover & $C=1$ & 1.3 & 99.24 & 11.54 & -88.46 \\
\hline
\end{tabular}

SetCover strategy with $C=10$. In terms of the recall and result similarity metrics, we observe very similar values. The metrics obtained by using the query $\log$ B2 (Table 4) confirm the validity of the results since they exhibit similar behavior.

Query coverage Coverage metrics are relatively high for all strategies (Table 4). The worst performing strategy (Summarization with $S=10$ ) results in $71 \%$ of unique test queries being covered. For the same strategy, the coverage goes up to almost $94 \%$ when the query volume is considered. Certain strategies attain the same coverage value attained by the full web index or very close values (e.g., Summarization with $S=160$ or the SetCover strategy). We observe similar behavior when query log B2 is used (Table 5). Since the aDCP heuristic uses the most popular documents, it is very successful, as expected, with query volume coverage, but still cannot beat the largest summarization index. The best volume coverage is for aDCP using $\mu=0.3$ with $96 \%$. On the other hand, aDCP is not as good for unique query coverage.

\subsection{Relevance}

To understand the relevance loss due to the pruned indexes, here we use the web collection A with our LTR ranking variant and the test queries from query log B1. Based on the results of the previous section, we analyze only the heuristics with higher precision score. For example, DocumentSize with $L=1 \%$.

We make an exception in the case of the Summarization heuristics, to understand better its effect in relevance. In total we try seven cases and we evaluate relevance using NDCG with respect to the full index version. We do not include stemming nor stopwords removal in this case, because the LTR variant has these functionality embedded during the feature extraction process.

The results for this experiment are given in Table 6 and Figure 2. For the summarization heuristics, the relevance loss is marginal, while for the other techniques there is a significant NDCG loss, from $20 \%$ to $89 \%$. We observe that NDCG loss is much higher for the heuristics that uses document pruning (aDCP and Set Cover). Nevertheless, this effect is also augmented by the fact that the relevance judgments do not cover the whole collection. 


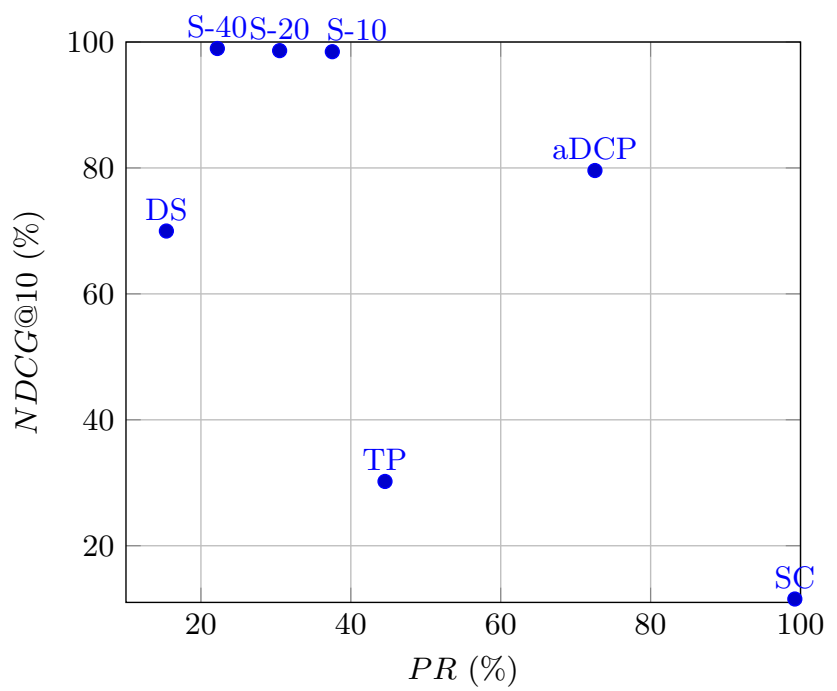

Fig. 2. Pruning ratio versus NDCG@10 (collection A, LTR, query log B1).

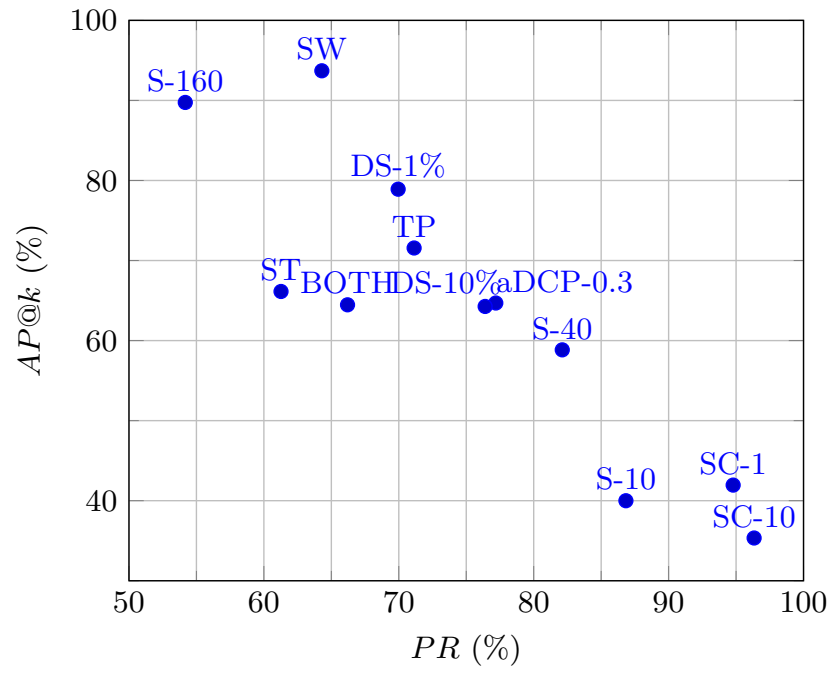

Fig. 3. Pruning ratio vs. precision (CC collection, BM25, query log A).

\subsection{Trade-off Analysis}

Figure 3 shows the trade-off between the pruning ratio and the precision attained by different pruning strategies. In general, as the pruning ratio increases the precision is observed to decrease, as expected. The Summarization strategy (with $S=160$ ) can be seen to cut the index size by half with around only $10 \%$ decrease in precision with respect to the full web index. Therefore, search engines, for which quality is vital, can use this technique to achieve drastic reduction in the hardware needed for storing and processing the web index. The SetCover 


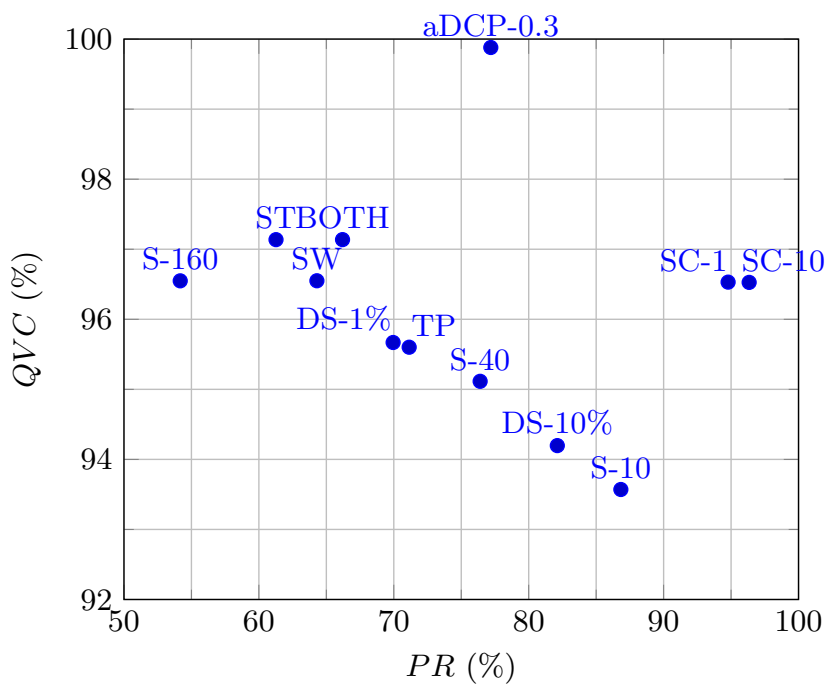

Fig. 4. Pruning ratio vs. query volume coverage (CC collection, BM25, query log A).

strategy, on the other hand, results in significant quality loss (around 60\%), but can yield huge resource savings (around 95\%). Therefore, commercial search engines that operate with very limited resources can employ these strategy to cut their operational costs.

Figure 4 shows a similar trade-off between the pruning ratio and query volume coverage. The Summarization strategy (with $S=160$ ) results in high query volume coverage, again, under the same pruning ratio. The SetCover strategy, however, achieves almost the same query volume coverage (around 96.5\%), with a drastic reduction in index size. Therefore, a search engine, which aims to satisfy as many queries as possible (rather than the aggregate performance over many queries) may adopt SetCover as its pruning strategy.

Regarding relevance, the summarization technique allows to keep parts of all the documents, leading to a marginal NDCG@10 drop, with a reasonable index size reduction. So clearly they are very competitive. The differences that appear in the pruning ratios for some heuristics, such as Summarization and Documentsize, can be explained by the fact that collection $\mathrm{A}$ is of better quality (that is, has much less web spam as has been curated) and also is more homogeneous and hence the fraction of documents removed diminishes.

One way to do a fair comparison of all the cases is to normalize by the index size in percentage. That is, compute the power factor gain of each measure dividing by the index size (the factor would be 1 for the full index in most cases). Larger the power factor, more you gain per space unit used. In Figure 5 we show these values for the average precision (maximum factor of almost 10), the query volume coverage (maximum factor over 26), and NDCG@10 (maximum factor over 15), using a log-log graph. In both cases, smaller the index, larger the power factor, which is expected given the power law shown in [4] (few documents 


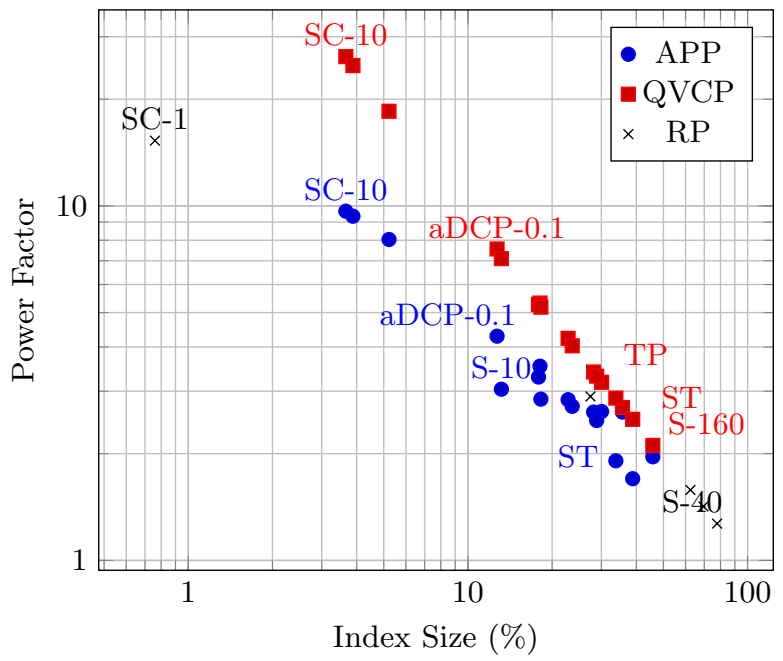

Fig. 5. Power factor for average precision, query volume coverage and NDCG@10.

answers many queries). In this sense, almost all the strategies are similar as there are almost no real outliers. One interesting fact is that the quality measures (APP and RP) are almost in a line while QVCP is almost a perfect line showing an underline power law for this power factor.

\section{Conclusions}

We have shown different index pruning strategies that aim to reduce the index size significantly, while keeping the search quality and query coverage as similar as possible to the case of the full web index. We conducted large-scale experiments demonstrating the feasibility of these approaches. Our results show that there is no clear dominant technique for different query sets and surely the same is true for different web document collections. We have not included the gains in query processing time, but in all the techniques we get significant faster processing times, from 50\% more throughput for S160 to almost a 10 times improvement for the most aggressive pruning techniques.

In practice, each search application has different use cases and different hardware resources. So, we believe that constructing an index that will satisfy all kinds of use cases is not feasible in practice. However, we can recommend different index pruning techniques for different use cases. For example, if we do not have large amounts of hardware and the quality of results is not critical, SetCover or Summarization with a small $S$ parameter (e.g., $S=10$ ) can be a good fit. If we have lots of hardware and search quality is vital, then the Summarization strategy with a large $S$ parameter $(e . g ., S=80)$ or the DocumentSize strategy with a small $L$ parameter (e.g., $L=1 \%$ ) might be good options. 


\section{References}

1. I. S. Altingovde, R. Ozcan, and O. Ulusoy. Static index pruning in web search engines: Combining term and document popularities with query views. ACM Trans. Inf. Syst., 30(1):2:1-2:28, 2012.

2. A. Anagnostopoulos, L. Becchetti, S. Leonardi, I. Mele, and P. Sankowski. Stochastic query covering. In Proceedings of the Fourth ACM International Conference on Web Search and Data Mining, WSDM '11, pages 725-734, New York, NY, USA, 2011. ACM.

3. J. Arguello, J. Callan, and F. Diaz. Classification-based resource selection. In Proceedings of the 18th ACM Conference on Information and Knowledge Management, pages 1277-1286, New York, NY, USA, 2009. ACM.

4. R. Baeza-Yates, P. Boldi, and F. Chierichetti. Essential web pages are easy to find. In Proceedings of the 24th International Conference on World Wide Web, WWW 2015, Florence, Italy, May 18-22, 2015, pages 97-107, 2015.

5. R. Baeza-Yates, A. Gionis, F. Junqueira, V. Murdock, V. Plachouras, and F. Silvestri. Design trade-offs for search engine caching. TWEB, 2(4):20:1-20:28, 2008.

6. R. Baeza-Yates, V. Murdock, and C. Hauff. Efficiency trade-offs in two-tier web search systems. In Proceedings of the 32nd International ACM SIGIR Conference on Research and Development in Information Retrieval, pages 163-170, New York, NY, USA, 2009. ACM.

7. R. Baeza-Yates and B. Ribeiro-Neto. Modern information retrieval: The concepts and technology behind search. Addison-Wesley, Pearson, 2011.

8. R. Blanco and A. Barreiro. Static pruning of terms in inverted files. In Proceedings of the 29th European Conference on IR Research, pages 64-75, Berlin, Heidelberg, 2007. Springer-Verlag.

9. R. Blanco and A. Barreiro. Probabilistic static pruning of inverted files. $A C M$ Trans. Inf. Syst., 28(1):1:1-1:33, 2010.

10. S. Büttcher and C. L. A. Clarke. A document-centric approach to static index pruning in text retrieval systems. In Proceedings of the 15th ACM International Conference on Information and Knowledge Management, pages 182-189, New York, NY, USA, 2006. ACM.

11. B. B. Cambazoglu and R. Baeza-Yates. Scalability challenges in web search engines. Morgan \& Claypool Publishers, 2015.

12. D. Carmel, D. Cohen, R. Fagin, E. Farchi, M. Herscovici, Y. S. Maarek, and A. Soffer. Static index pruning for information retrieval systems. In Proceedings of the 24th Annual International ACM SIGIR Conference on Research and Development in Information Retrieval, pages 43-50, New York, NY, USA, 2001. ACM.

13. R.-C. Chen and C.-J. Lee. An information-theoretic account of static index pruning. In Proceedings of the 36th International ACM SIGIR Conference on Research and Development in Information Retrieval, pages 163-172, New York, NY, USA, 2013. ACM.

14. E. S. de Moura, C. F. dos Santos, D. R. Fernandes, A. S. Silva, P. Calado, and M. A. Nascimento. Improving web search efficiency via a locality based static pruning method. In Proceedings of the 14th International Conference on World Wide Web, pages 235-244, New York, NY, USA, 2005. ACM.

15. A. Kulkarni, A. S. Tigelaar, D. Hiemstra, and J. Callan. Shard ranking and cutoff estimation for topically partitioned collections. In Proceedings of the 21st ACM International Conference on Information and Knowledge Management, pages 555564, New York, NY, USA, 2012. ACM. 
16. G. Leung, N. Quadrianto, K. Tsioutsiouliklis, and A. J. Smola. Optimal web-scale tiering as a flow problem. In J. Lafferty, C. Williams, J. Shawe-Taylor, R. Zemel, and A. Culotta, editors, Advances in Neural Information Processing Systems 23, pages 1333-1341. Curran Associates, Inc., 2010.

17. A. Ntoulas and J. Cho. Pruning policies for two-tiered inverted index with correctness guarantee. In Proceedings of the 30th Annual International ACM SIGIR Conference on Research and Development in Information Retrieval, pages 191-198, New York, NY, USA, 2007. ACM.

18. D. Puppin, F. Silvestri, R. Perego, and R. Baeza-Yates. Tuning the capacity of search engines: Load-driven routing and incremental caching to reduce and balance the load. ACM Transactions on Information Systems, 28(2):1-36, 2010.

19. K. M. Risvik, Y. Aasheim, and M. Lidal. Multi-tier architecture for web search engines. In Proceedings of the 1st Conference on Latin American Web Congress, page 132, Washington, DC, USA, 2003. IEEE Computer Society.

20. G. Skobeltsyn, F. Junqueira, V. Plachouras, and R. Baeza-Yates. ResIn: a combination of results caching and index pruning for high-performance web search engines. In Proceedings of the 31st Annual International ACM SIGIR Conference on Research and Development in Information Retrieval, pages 131-138, New York, NY, USA, 2008. ACM.

21. S. L. Thota and B. Carterette. Within-document term-based index pruning with statistical hypothesis testing. In Proceedings of the 33rd European Conference on Advances in Information Retrieval, pages 543-554, Berlin, Heidelberg, 2011. Springer-Verlag.

22. Q. Wu, C. J. C. Burges, K. M. Svore, and J. Gao. Adapting boosting for information retrieval measures. Information Retrieval, 13(3):254-270, Jun 2010. 